\title{
Mikroarrays bestehend aus pH-sensitiven Polymeren als Sonden für Sensoren basierend auf Oberflächenplasmonenresonanz
}

\author{
Alfred Kick ${ }^{1}$, Michael Mertig ${ }^{1,2}$ \\ ${ }^{1}$ Kurt-Schwabe-Institut für Mess- und Sensortechnik e.V. Meinsberg, Kurt-Schwabe-Straße 4, 04736 \\ Waldheim, Deutschland \\ kick@ksi-meinsberg.de \\ ${ }^{2}$ Professur für Physikalische Chemie, Mess- und Sensortechnik, Technische Universität Dresden, \\ 01069 Dresden, Deutschland
}

\begin{abstract}
Zusammenfassung:
In dieser Arbeit wird die Herstellung eines Polymer-Mikroarray vorgestellt. Die dazu verwendeten Lösungen der $\mathrm{pH}$-sensitiven Polyvinylpyridine werden auf einer reaktiv beschichteten Goldoberfläche durch ein Mikropipettiersystem tropfenweise in Abständen von $290 \mu \mathrm{m}$ auf 20 verschiedenen Messorten abgesetzt. Mittels der Methode der Oberflächenplasmonenresonanz (SPR) werden pH-WertÄnderungen in sauren, wässrigen Lösungen auf dem Mikroarray detektiert. Die SPR-Methode erlaubt eine hochempfindliche und markierungsfrei optische Bestimmung der Brechungsindexänderung. Das Quellen und die Protonierung der Polymere führen zu messbaren Brechungsindexänderungen an der Sensoroberfläche. Das Mikroarray wird in einem mikrofluidischen Kanal eingesetzt, um pH-Werte kleiner als 5 in salzsauren Lösungen mittels SPR schnell zu detektieren. Jedes der Polymere zeigt einen bestimmten $\mathrm{pH}$-Bereich, in dem es die höchste Sensitivität aufweist.
\end{abstract}

Schlagwörter: Oberflächenplasmonenresonanz, pH-Sensor, pH-sensitiv, Polymer, Mikroarray.

\section{Einleitung}

Das Ziel der vorgestellten Arbeit ist die Entwicklung eines Verfahrens zur Herstellung eines Polymer-Mikroarrays, welches die optische $\mathrm{pH}$ Bestimmung in sauren wässrigen Lösungen ermöglicht. Das Sensorprinzip beruht dabei auf der Oberflächenplasmonenresonanz (SPR). Die SPR-Methode erlaubt eine sehr sensitive optische und markierungsfreie Messung der Brechungsindexänderung an einer etwa $50 \mathrm{~nm}$ dicken Goldschicht [1]. Um dieses Prinzip nutzen zu können, müssen $\mathrm{pH}$-sensitive Polymere auf der Goldoberfläche des SPR-Sensors immobilisiert werden. Dafür sind Polymere mit basischen Gruppen als pH-Sonden geeignet. Hier werden Polyvinylpyridine verwendet, welche über eine Poly(glycidylmethacrylat)-Schicht an die Goldoberfläche gebunden werden (Bild 1). In sauren Lösungen werden Pyridingruppen protoniert. Diese Protonierung und das Quellen der Polymerschichten hat eine Änderung des Brechungsindex an der Sensoroberfläche zur Folge. Auf dieser Grundlage soll ein optischer $\mathrm{pH}$-Sensor entwickelt werden, welcher ohne konventionelle $\mathrm{pH}$-Elektroden betrieben werden kann (Bild 2).<smiles>[X]C(CC(C)(C)C)CC(C)(C)C</smiles>

P4VP<smiles>[R]C(C)(C)CC(c1ccccn1)C(C)(C)C</smiles>

P2VP-R

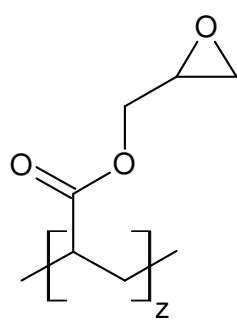

PGMA
Bild 1. Chemische Struktur ausgewählter Polymere. P4VP - Poly(4-vinylpyridin), P2VP-R - Poly(2vinylpyridin) mit einem terminalen Rest $R(R=H$, $\mathrm{COOH}, \mathrm{SH}), \mathrm{PGMA}$ - Poly(glycidylmethacrylat).

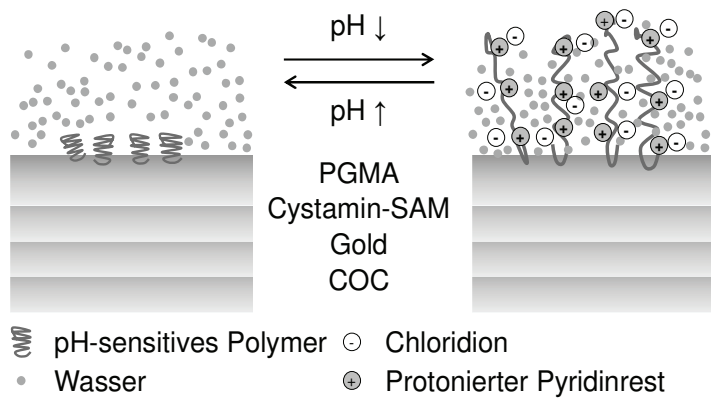

Bild 2. Aufbau der Sensorschicht und Prinzip der $\mathrm{pH}$-Antwort in HCl-Lösungen. Mit der Protonierung der Pyridingruppen werden Wasser und Chloridionen in der gequollenen Polymerschicht gebunden. 
Dadurch ergeben sich wesentliche Vorteile gegenüber den elektrochemischen Methoden. Diese Vorteile betreffen beispielsweise die Miniaturisierung sowie die Messung bei extremen $\mathrm{pH}$-Werten und bei geringen lonenstärken der Probenlösungen [2]. Weiterhin wird ein mikrofluidischer Kanal verwendet, um die Messung zu beschleunigen und um das gebrauchte Probenvolumen deutlich unter $1 \mathrm{ml} z u$ halten. Das verwendete SPR-Spektrometer erlaubt die Auswertung eines Mikroarrays unterschiedlicher Polymere mit Sensitivitäten in spezifischen $\mathrm{pH}$-Bereichen. Dadurch kann in einem weiten $\mathrm{pH}$-Bereich gemessen werden.

\section{Material und Methoden}

\section{SPR-Chip und SPR-Spektrometer}

Der Aufbau des verwendeten SPR-Systems (capitalis technology $\mathrm{GmbH}$, Germany) wurde bereits in früheren Publikationen beschrieben $[3,4]$. Diese Plattform verwendet transparente Chips $(76 \mathrm{~mm} \times 26 \mathrm{~mm} \times 4 \mathrm{~mm})$ aus einem Cyclo-Olefin-Copolymer (COC) mit integrierten Mikrolinsen und einer $50-\mathrm{nm}$ dicken Goldschicht mit einer effektiven Sensorfläche von $3 \mathrm{~mm} \times 9 \mathrm{~mm}$. Ein eindimensional aufgelöstes Array von streifenförmigen Sondenorten der Sensorfläche wird ausgewertet. Die Verschiebungen der Reflektivitätsminima im winkelaufgelösten Spektrum werden als SPRSignale aufgezeichnet. Die SPR-Signale, angegeben in Pixel einer CCD-Kamera, sind die Verschiebungen der Minima des reflektierten Lichtes im Winkelspektrum. Die Lage dieser Minima wird simultan als Mittelwert über die Länge eines streifenförmigen Sondenortes registriert. Als Mikrokanal wird ein temperierbares Mikrofluidik-System mit einem Mikrokanal aus Polydimethylsiloxan verwendet. Dieser Kanal ist $300 \mu \mathrm{m}$ hoch und $3 \mathrm{~mm}$ breit. Die Flüssigkeiten werden mittels einer Spritzenpumpe durch den Mikrokanal über die Sensoroberfläche gepumpt.

\section{Immobilisierung der Polymere}

Die Goldschicht des SPR-Sensors wird durch Überschichten mit einer wässrigen Lösung von $10 \mathrm{mM}$ Cystamindihydrochlorid funktionalisiert. Cystamin bindet über die Disulfidgruppe an Gold und stellt Aminogruppen zur Verfügung, indem es eine selbstorganisierende Monoschicht (SAM) bildet. Eine Schicht, bestehend aus Poly(glycidylmethacrylat) mit einer durchschnittlichen Molmasse von $18 \mathrm{~kg} / \mathrm{mol}$ (PGMA), wird auf der Cystamin-SAM immobilisiert, wobei die Glycidylgruppen des PGMA mit den Aminogruppen des SAM reagieren können. Dazu werden $40 \mu$ leiner $10 \mathrm{~g} / \mathrm{l}$ PGMA-Lösung in Aceton auf die Cystamin-SAM pipettiert und $1 \mathrm{~h}$ bei $110^{\circ} \mathrm{C}$ im Vakuumofen inkubiert und unge- bundenes PGMA wird danach durch Spülen mit Aceton entfernt.

Die Polymere aus Tabelle 1 werden durch ein Mikropipettiersystem (sciFLEXARRAYER S3, SCIENION AG) auf die PGMA-Schicht aufgetragen. Dabei werden Tropfen mit einem Volumen von etwa $100 \mathrm{pl}$ abgesetzt.

Tabelle 1: pH-sensitive Polymere auf dem Mikroarray. M - Massenmittel der Molmasse in $\mathrm{kg} / \mathrm{mol}$.

\begin{tabular}{|c|c|c|}
\hline Polymer & Abkürzung & M \\
\hline $\begin{array}{c}\text { Verzweigtes Polyethy- } \\
\text { lenimin }\end{array}$ & PEI & 25 \\
\hline Poly(4-vinylpyridin) & P4VP 60 kDa & 60 \\
\hline $\begin{array}{c}\text { Poly(2-vinylpyridin) mit } \\
\text { terminaler Thiolgruppe }\end{array}$ & $\begin{array}{c}\text { P2VP-SH } \\
3 \mathrm{kDa}\end{array}$ & 3 \\
\hline $\begin{array}{c}\text { Poly(2-vinylpyridin) mit } \\
\text { terminaler Car- } \\
\text { boxylgruppe }\end{array}$ & $\begin{array}{c}\text { P2VP-COOH } \\
41 \mathrm{kDa}\end{array}$ & 41 \\
\hline Poly(2-vinylpyridin) & P2VP 41 kDa & 41 \\
\hline Poly(2-vinylpyridin) & P2VP $38 \mathrm{kDa}$ & 38 \\
\hline
\end{tabular}

Die $\mathrm{pH}$-sensitiven Polymere liegen in einer wässrigen Lösung mit $10 \%$ Ethanol und $1 \mathrm{~g} / \mathrm{l}$ Polymer vor. Die Tropfenerzeugung in den piezogesteuerten Kapillaren wird durch Variation der Spannung und Pulsdauer optimiert (Bild 3). Die optimierte Spannung beträgt $71 \mathrm{~V}$ und die Pulsdauer $56 \mu$ s.
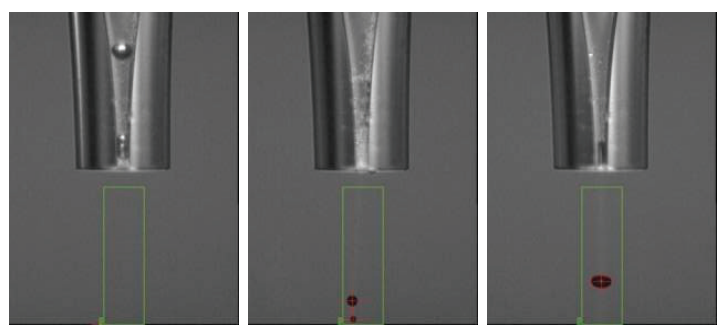

Bild 3. Optimierung der Parameter zur Abgabe der Polymerlösungen. Links: Spannung und Pulsdauer sind zu klein; Gasbläschen bilden sich; keine Tropfenbildung. Mitte: Spannung zu hoch; mehrere Tropfen mit schlecht definierter Flugbahn. Rechts: Symmetrischer Tropfen: $\approx 100$ pl, Spannung: 71 V, Pulsdauer: $56 \mu$ s.

Weil die Polymere gerätebedingt in streifenförmigen Sondenorten aufgebracht werden müssen, wird die Immobilisierung in zwei Durchläufen realisiert. Zunächst werden die Tropfen in einem Abstand von $80 \mu \mathrm{m}$ innerhalb eines Sondenortes abgesetzt. Anschließend wird im zweiten Durchlauf um $40 \mu \mathrm{m}$ versetzt, um mögliche Lücken zu schließen. Der Abstand zwischen den Sondenstreifen beträgt $290 \mu \mathrm{m}$. Es werden 7 unterschiedliche Lösungen im Mikroarray in vier Blöcken von links nach rechts aufgetragen: 
1) $10 \%$ Ethanol in Wasser, 2) PEI, 3) P4VP 60 $\mathrm{kDa}$, 4) P2VP-SH $3 \mathrm{kDa}$, 5) P2VP-COOH $41 \mathrm{kDa}, 6)$ P2VP $41 \mathrm{kDa}, 7)$ P2VP 38 kDa. Auf dem ersten Block sind je Pipettierposition 1, auf dem zweiten 2, auf dem dritten 3 und auf dem vierten 4 Tropfen der jeweiligen Lösung abgesetzt (Bild 4). Anschließend wird das Mikroarray 30 min bei $110{ }^{\circ} \mathrm{C}$ im Vakuumofen inkubiert.

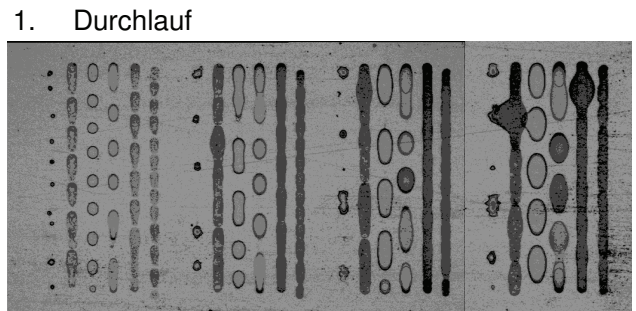

2. Durchlauf

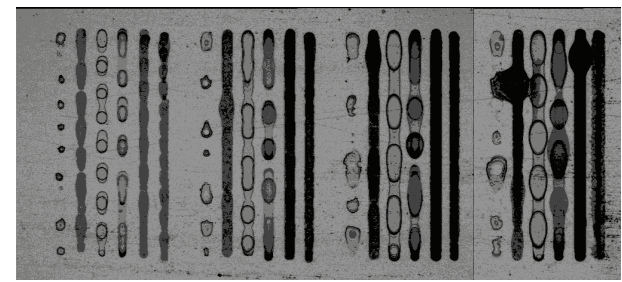

Bild 4. Aufbaus des Polymer-Mikroarrays mittels eines Mikropipettiersystems. Sichtbar sind $4 \times 6$ vertikale streifenförmige Rückstände der Polymerlösungen; von links nach rechts: 1) $10 \%$ Ethanol in Wasser (keine sichtbaren Rückstände), 2) PEl, 3) P4VP $60 \mathrm{kDa}$, 4) P2VP-SH $3 \mathrm{kDa}$, 5) P2VP-COOH $41 \mathrm{kDa}, 6)$ P2VP $41 \mathrm{kDa}$, 7) P2VP $38 \mathrm{kDa}$. Tropfenanzahl pro Position und Block von links nach rechts: 1, 2, 3 bzw. 4 Tropfen. Abstand der Tropfenposition innerhalb der Streifen: $80 \mu \mathrm{m}$ (37 Tropfenpositionen), zwischen unterschiedlichen Streifen $290 \mu \mathrm{m}$. Im zweiten Durchlauf wird der Absatzpunkt um $40 \mu \mathrm{m}$ vertikal versetzt.

\section{SPR-Messung}

Zu Beginn der Messung wird der SPR-Chip mit einer wässrigen Lösung von $100 \mathrm{mM} \mathrm{HCl}$ gespült, um ungebundene Polymere zu entfernen und ein stabiles SPR-Signal zu erhalten. Anschließend werden Lösungen mit unterschiedli-

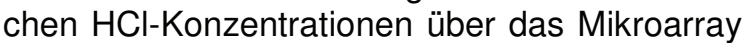
gepumpt. Die pH-Werte liegen zwischen 0,75 und 4,20. Das Probenvolumen beträgt $200 \mu \mathrm{l}$ und die Flussrate 7,7 $\mu \mathrm{l} / \mathrm{s}$. Die SPR-Signale bei $25^{\circ} \mathrm{C}$ werden für jede Lösung über einen Zeitraum von 2 min gemittelt. Die Referenzierung der Signale erfolgt durch Subtraktion der Signale, die von Referenzsondenorten stammen, auf denen PGMA ohne $\mathrm{pH}$-sensitive Polymere immobilisiert ist.

\section{Ergebnisse und Diskussion}

Eine stabile Immobilisierung der $\mathrm{pH}$-sensitiven Polymere auf der Goldoberfläche eines SPRChips wurde durch eine vorherige reaktive Funktionalisierung gewährleistet. Dies konnte anhand der gut reproduzierbaren und stabilen SPR-Signale verifiziert werden. Die chemische Anbindung des PGMA und der Polyvinylpyridine war somit erfolgreich. Welche chemische Reaktion dabei stattfindet, konnte nicht direkt nachgewiesen werden. Aus der Literatur ist jedoch bekannt, dass sowohl Amino-, Thiolund Carboxylgruppen als auch Pyridin-Reste bei höheren Temperaturen mit Glycidylgruppen reagieren können $[5,6]$.

Um Polymere in einem Mikroarray anzuordnen, wurden zunächst die notwendigen Parameter für die Tropfenabgabe mittels piezoelektrisch gesteuerter Kapillarspitzen bestimmt (Spannung: $71 \mathrm{~V}$, Pulsdauer: $56 \mu \mathrm{s})$. Diese Tropfen haben unterschiedliche Kontaktwinkel auf der Chipoberfläche und ergeben dadurch unterschiedliche Kontaktdurchmesser. In Bild 4 ist erkennbar, dass, obwohl 37 Tropfen im ersten Durchlauf und Block abgesetzt wurden, deutlich weniger getrennte Bereiche der Rückstände innerhalb eines Streifens feststellbar sind. Dieses lässt sich dadurch erklären, dass der Kontaktdurchmesser der Tropfen größer ist als der Abstand der Tropfenpositionen von $80 \mu \mathrm{m}$. Somit vereinigen sich die Tropfen während des Pipettierens. Wie mobil die Tropfen auf der Oberfläche sind, wird vor allem durch die Viskosität der Lösung, die Benetzbarkeit auf der Oberfläche und daher durch das gelöste Polymer bestimmt. Folglich ergibt sich für eine bestimmte Polymerlösung eine charakteristische Oberflächenmorphologie der Rückstände. So können die Polymere P4VP 60 kDa, P2VP $41 \mathrm{kDa}$ und P2VP $38 \mathrm{kDa}$ gut zur Herstellung streifenförmiger Sondenorte genutzt werden. PEI ist unter den getesteten Bedingungen ungeeignet und konnte keine nutzbaren SPRSignale liefern. Die abgesetzte Polymermenge ist ebenfalls ein wichtiger Parameter. Das wird dadurch bestätigt, dass gut auswertbare Signale der anderen 5 Polymere vom dritten Block mit 3 Tropfen pro Position erhalten werden. Die entsprechenden SPR-Signale in Abhängigkeit vom pH-Wert sind in Bild 5 dargestellt. Auf allen Sondenorten sinkt das SPR-Signal mit steigendem pH-Wert, außer auf dem von P2VP 41 kDa. Auf der Schicht von P2VP $41 \mathrm{kDa}$ läuft das SPR-Signal durch ein Minimum bei $\mathrm{pH} \approx 3$ und das Signal ist zwischen $\mathrm{pH} 2,3$ und 3,8 negativ. Daher kann man von einer Verdünnung bzw. einem Quellen der Polymerschicht ausgehen. Das führt zur Erniedrigung des effektiven Brechungsindex an der Sensoroberfläche und damit zu geringeren SPR-Signalen. Der Signalanstieg bei weiterer Erhöhung der $\mathrm{HCl}-K o n z e n t r a t i o n$ und damit einer Senkung des $\mathrm{pH}-$ Wertes lässt sich durch die Einlagerung des $\mathrm{HCl}$ in der Polymerschicht erklären. Die Erhöhung der $\mathrm{HCl}$-Konzentration führt zu höhe- 
ren Brechungsindices und höheren SPRSignalen.

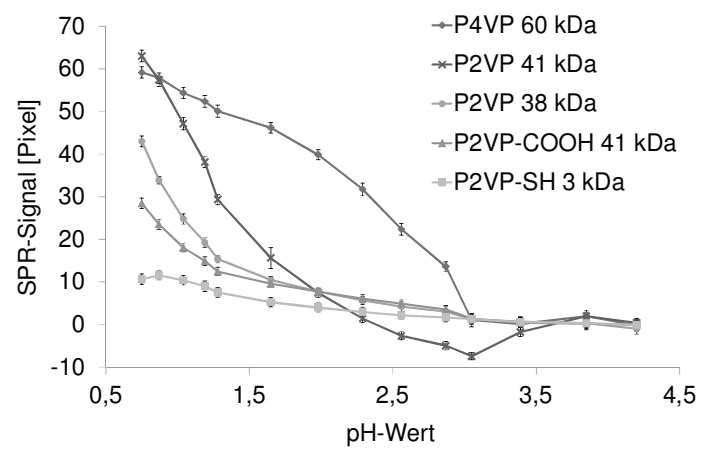

Bild 5. SPR-Signale in Abhängigkeit vom pH-Wert einer $\mathrm{HCl}$-Lösung auf verschiedenen Polymerschichten des Mikroarrays (3. Block von links, Bild 4).

Die pH-Sensitivität, in Form des Signalanstiegs

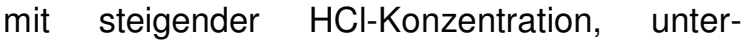
scheidet sich zwischen den Polymeren. P4VP 60 kDa und P2VP 41 kDa mit den größten Molmassen zeigen hohe SPR-Signale und einen großen sensitiven Bereich von $\mathrm{pH} \mathrm{0,75}$ bis 3. Schichten mit P2VP 41 kDa zeigen eine höhere Steilheit der $\mathrm{pH}$-abhängigen SPR-Signale. Auf Messflächen mit P2VP-COOH $41 \mathrm{kDa}$ ist der $\mathrm{pH}$-sensitive Bereich zu niedrigeren $\mathrm{pH}$ Werten verschoben, was auf eine veränderte Struktur der Schichten oder möglicherweise den Einfluss des sauren Charakters der Carboxylgruppe hinweist. Dies zeigt sich auch im Vergleich mit Schichten des P2VP 38 kDa mit einer etwas niedrigeren Molmasse. Bei der wesentlich geringeren Molmasse von P2VP-SH $3 \mathrm{kDa}$ sind die SPR-Signale entsprechend viel geringer, weil davon ausgegangen werden kann, dass weniger $\mathrm{HCl}$ durch die Schicht aufgenommen wird [7], da die effektive Konzentration der protonierbaren Pyridingruppen an der Sensoroberfläche geringer ist. Dies beeinflusst auch die Lage des pH-sensitiven Bereiches, welcher von der Zugänglichkeit der Pyridingruppen abhängt. Bei besser zugänglichen Gruppen, wie z. B. bei P4VP 60 kDa im Vergleich zu P2VP $41 \mathrm{kDa}$, verschiebt sich der $\mathrm{pH}$ sensitive Bereich zu höheren Werten [2].

\section{Schlussfolgerungen und Ausblick}

Es wurde ein Verfahren entwickelt, mit der es möglich ist, Polymer-Mikroarrays auf Goldoberflächen herzustellen. Dabei wurden Polymerlösungen tropfenweise in Abständen von $290 \mu \mathrm{m}$ auf gesonderten Messorten mit einem Mikropipettiersystem abgesetzt. Mit den getesteten $\mathrm{pH}$ sensitiven Polymeren, den Polyvinylpyridinen mit unterschiedlichen Molmassen und terminalen funktionellen Gruppen, wurden Messungen auf SPR-Chips durchgeführt. Es wurde gezeigt, dass die $\mathrm{pH}$-Sensitivität auf Schichten mit höhe- ren Molmassen größer ist, als bei Schichten mit Polymeren geringerer Molmasse. Dabei sind die Protonierung und die $\mathrm{HCl}$-Aufnahme in den Polymerschichten entscheidend für die Höhe der gemessenen SPR-Signale. Außerdem beeinflusst die chemische Struktur eines Polymers die SPR-Signalhöhe und die Lage des $\mathrm{pH}$ sensitiven Bereiches. Das hergestellte Mikroarray zeigt eine $\mathrm{pH}$-Sensitivität über einen weiten $\mathrm{pH}$-Bereich in $\mathrm{HCl}$-Lösungen von $\mathrm{pH} \mathrm{0,75}$ bis pH 3. Zukünftig soll die Immobilisierung der Polymere noch homogener gestaltet werden sowie Polymere mit anderen basischen und sauren Gruppen sollen getestet werden.

\section{Danksagung}

Diese Arbeit wurden durch das Sächsische Ministerium für Wissenschaft und Kunst (Förderkennzeichen: 4-7531.50/1147/2-2014) gefördert.

\section{Literatur}

[1] J. Homola, S. Yee, G. Gauglitz, Surface Plasmon Resonance Sensors: Review, Sensors and Actuators B: Chemical 54 (1-2), 3-15 (1999); doi: 10.1016/S0925-4005(98)00321-9

[2] A. Kick, M. Mertig, Characterization of pHsensitive Polymer Layers by Surface Plasmon Resonance and Quartz Crystal Microbalance, Physica Status Solidi A 212 (6), 1862-6319 (2015); doi: 10.1002/pssa.201431604

[3] N. Danz, A. Hofmann, Plasmon Resonance Sensor, WO 2008/025488

[4] N. Danz, A. Kick, F. Sonntag, S. Schmieder, B. Höfer, U. Klotzbach, M. Mertig, Surface Plasmon Resonance Platform Technology for MultiParameter Analyses on Polymer Chips, Engineering in Life Sciences 11 (6), 566-572 (2011); doi: 10.1002/elsc.201000192

[5] I. Tokareva, S. Minko, J. H. Fendler, E. Hutter, Nanosensors Based on Responsive Polymer Brushes and Gold Nanoparticle Enhanced Transmission Surface Plasmon Resonance Spectroscopy, Journal of the American Chemical Society 126 (49), 15950-15951 (2004); doi: $10.1021 / \mathrm{ja} 044575 y$

[6] G. Xue, H. Ishida, J. L. Koenig, Chemical Reactions of Poly(vinyl Pyridine)s with epoxy compounds, Die Angewandte Makromolekulare Chemie 142 (1), 17-27 (1986); doi: 10.1002/apmc. 1986.051420102

[7] N. Tantavichet, M. D. Pritzker, C. M. Burns, Proton Uptake by Poly(2-Vinylpyridine) coatings, Journal of Applied Polymer Science 81 (6), 14931497 (2001); doi: 10.1002/app.1577 